\title{
Fingerprint of dynamical spin-charge correlations in the tunneling spectra of colossal magnetoresistive manganites
}

\author{
Silvia S. Seiro, ${ }^{*}$ Yanina Fasano ${ }^{\dagger}$ Ivan Maggio-Aprile, Edmond Koller, Rolf Lortz ${ }^{\ddagger}$ and $\varnothing y$ stein Fischer \\ Département de Physique de la Matière Condensée, Université de Genève, Quai Ernest-Ansermet 24, 1211 Geneva, Switzerland
}

(Received 31 October 2008; revised manuscript received 14 April 2009; published 17 September 2009)

\begin{abstract}
We present temperature-dependent scanning tunneling spectroscopy measurements on $\mathrm{La}_{1-x} \mathrm{Ca}_{x} \mathrm{MnO}_{3}(x$ $\sim 0.33$ ) films with different degrees of biaxial strain. A depletion in normalized conductance around the Fermi level is observed both above and below the insulator-to-metal transition temperature $T_{\mathrm{MI}}$, for weakly as well as highly strained films. This pseudogaplike depletion globally narrows on cooling. The zero-bias conductance decreases on cooling in the insulating phase, reaches a minimum close to $T_{\mathrm{MI}}$ and increases on cooling in the metallic phase, following the trend of macroscopic conductivity. These results support a recently proposed scenario in which dynamical short-range antiferromagnetic/charge order correlations play a preeminent role in the transport properties of colossal magnetoresistive manganites [R. Yu et al., Phys. Rev. B 77, 214434 (2008)].
\end{abstract}

DOI: 10.1103/PhysRevB.80.100404

PACS number(s): 75.47.Lx, 71.30.+h, 68.37.Ef, 73.50.-h

In spite of extensive theoretical and experimental research on colossal magnetoresistive manganites, the mechanism underlying the transition from insulator to metal-like transport concomitant to ferromagnetic ordering has not yet been completely understood. ${ }^{1}$ In the paramagnetic (PM) phase, tunneling spectroscopic measurements are in agreement with the presence of a gap in the density of states: Tunneling conductance presents a depletion at low bias voltages ${ }^{2,3}$ and the zero-bias conductance follows a thermally activatedlike behavior. $^{3,4}$ On cooling into the ferromagnetic state the depletion does not disappear, ${ }^{2,3}$ in apparent contrast to the macroscopic transport properties. In this work we show that for $\mathrm{La}_{0.7} \mathrm{Ca}_{0.3} \mathrm{MnO}_{3}$ (LCMO) films of different strain levels the depletion observed in both the insulating and metallic regimes is accompanied by a spectral weight redistribution on cooling through $T_{\mathrm{MI}}$. The temperature evolution of the zero-bias conductance (ZBC) accounts for the macroscopic insulator-to-metal transition. Our results support a recent theoretical study which shows that dynamical nanoscale antiferromagnetic/charge order (AFM/CO) correlations give rise to a pseudogap in the density of states (DOS) around the chemical potential $(\mu)$, not only above but also below the insulator-to-metal transition temperature $T_{\mathrm{MI}}{ }^{5}$

The films studied in this work have been grown by $\mathrm{rf}$ sputtering on (100) $\mathrm{SrTiO}_{3} \quad(\mathrm{STO})$ and (110) $\mathrm{NdGaO}_{3}$ (NGO). The growth procedure has been reported in detail in Refs. 2 and 6. X-ray diffraction measurements confirmed structural homogeneity, single crystallinity, and the presence of a single phase. Both LCMO/STO and LCMO/NGO films were found to be under strain. Reciprocal space mapping for LCMO/STO films confirmed an in-plane parameter equal to that of the substrate and a strongly reduced out-of-plane lattice parameter $(c \sim 3.80 \AA){ }^{2}$ The LCMO/NGO film is weakly strained $(c \sim 3.87 \AA)$ and compressed on average in the plane. ${ }^{6}$ As estimated from Laue oscillations, the thickness of the films is well above the dead-layer thickness. ${ }^{7,8}$

The resistivity $(\rho)$ of the films was measured in a fourpoint configuration, with the current flowing parallel to the film plane, along one of the main pseudocubic axis. Both kind of films exhibit a transition from insulator-to metal-like behavior at a temperature $T_{\mathrm{MI}}$ lower than that of bulk compounds, as expected for films under biaxial strain. ${ }^{6,7,9-12}$ For LCMO/STO, $T_{\mathrm{MI}}=154 \mathrm{~K}$ while for $\mathrm{LCMO} / \mathrm{NGO}, T_{\mathrm{MI}}$ $=235 \mathrm{~K}$. The residual resistivities are 5 and $0.35 \mathrm{~m} \Omega \mathrm{cm}$, respectively. By taking advantage of the shift in $T_{\mathrm{MI}}$ due to substrate-induced strain, ${ }^{6,7,9-12}$ we accessed the metallic and insulating phases in a wide range of temperatures.

Since three-dimensional perovskites lack an easy cleaving plane, we thoroughly cleaned the surface of the films with isopropanol in an ultrasonic bath prior to entering the sample in a variable-temperature home-made scanning tunneling microscope. This procedure has been widely applied to noncleavable manganites. , $^{23,14}$ Topographs and current vs voltage, $I(V)$, maps were measured as a function of temperature. Topographs were acquired at a constant current of $0.2-0.6$ $\mathrm{nA}$ and a bias voltage of 1-3 V. Spectroscopy was measured at a fixed tip-sample separation $(0.5 \mathrm{nA}$ at $0.5 \mathrm{~V}$ bias $)$ by recording the current when ramping the bias voltage. The tip electrode made of electrochemically etched Ir was grounded and the bias voltage $V$ was applied to the sample.

Topographs reveal flat terraces separated by growth steps multiple of the out-of-plane pseudocubic lattice parameter for both LCMO/NGO and LCMO/STO films. ${ }^{2,15}$ In Fig. 1(a) we show a typical topograph for the $42 \mathrm{~nm}$ thick LCMO/ NGO film. ZBC maps over an area covering a few topographic steps exhibit some dispersion of conductance values but no particular spatial pattern, as shown in Fig. 1(b) for LCMO/NGO and in Refs. 15 and 16 for LCMO/STO. For both LCMO/NGO and LCMO/STO films the ZBC distribution is Gaussian at all temperatures, complementing our previous results at higher energies on LCMO/STO films. ${ }^{2,15}$ Note that the ZBC level of a pixel in Fig. 1(b) is not the average of $\mathrm{ZBC}$ values over the pixel surface, but the point value at the pixel center. If there were sub-pixel regions of very high and very low $\mathrm{ZBC}$, as observed for Pr-doped LCMO films at $1.5-2 \mathrm{eV}$ energies, ${ }^{17}$ they should be manifest in the distribution of conductance values with a statistical weight proportional to their area fraction. The absence of a bimodal distribution of conductance values rather appears to indicate that there is no static phase separation in regions 

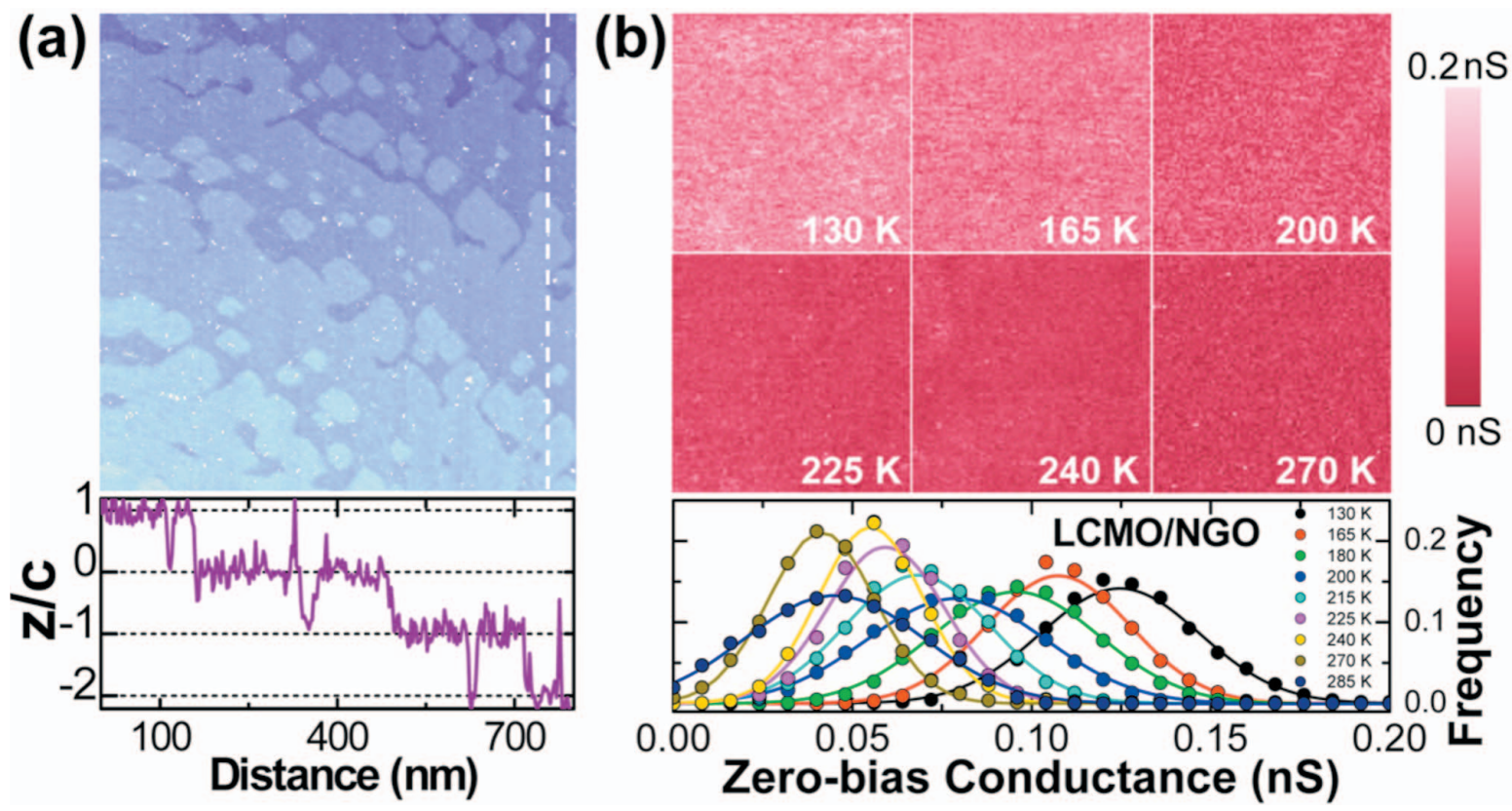

FIG. 1. (Color) Topographic and spectroscopic properties of weakly strained LCMO/NGO. (a) Top: $800 \times 800 \mathrm{~nm}^{2}$ topograph taken at $258 \mathrm{~K}$ for a bias voltage of $1.5 \mathrm{~V}$ and a tunnel current of $0.2 \mathrm{nA}$. Bottom: topographic profile along the dashed line. (b) Top: 450 $\times 450 \mathrm{~nm}^{2}$ zero-bias conductance maps taken at different temperatures for a junction impedance of $1 \mathrm{G} \Omega(0.5 \mathrm{~V} ; 0.5 \mathrm{nA})$. The color scale used is the same for all maps. Bottom: distribution of zero-bias conductance values. The full lines are Gaussian fits to the experimental distribution.

with strongly differentiated electronic properties. Assuming that a "different" phase has been missed by the discrete positioning of the tip, the surface fraction of it would be limited to roughly $10^{-4}$, casting a doubt on the role it plays in transport properties. The absence of regions with strongly differentiated tunneling spectroscopic properties (i.e., of a bimodal ZBC distribution) on LCMO films has also been reported for LCMO/NGO at $T_{\mathrm{MI}}$ (Ref. 18) and for cation ordered LCMO/ $\mathrm{MgO}(x=1 / 4)$ at 115 and $294 \mathrm{~K}^{13}$

For both strain states, not only the ZBC values but also the whole $I(V)$ curves at any given temperature display no bimodal distribution over the field of view. As an example, Fig. 2(a) shows local LCMO/STO $I(V)$ curves acquired over a $350 \mathrm{~nm}$-long path at temperatures below and above the transition. The current vs voltage curves in the metallic and insulating phases present a highly nonlinear character and are remarkably alike. At first sight, one might be tempted to link these observations to insulating behavior $[\operatorname{DOS}(\mu)=0]$. However, nonlinear $I(V)$ characteristics can appear also in metals $[\operatorname{DOS}(\mu) \neq 0]$, in particular if the DOS is nonconstant in the vicinity of $\mu$, or if the tunneling barrier is energydependent (e.g., for bias voltages that are a non-negligible fraction of the apparent work function ${ }^{19}$ ). Further information on the $\operatorname{DOS}(\mu)$ can be gained by a close inspection of the behavior of zero-bias conductance as a function of temperature.

Figure 2(b) presents the temperature evolution of macroscopic resistivity and the inverse of the ZBC obtained from the spatially averaged local $I(V)$ curves, for both weakly and highly strained films. We found that $1 / \mathrm{ZBC}$ is finite at all measured temperatures and roughly follows the behavior of macroscopic resistivity, increasing on warming in the low- temperature phase and decreasing on warming in the hightemperature phase. For a gapped DOS, a thermally activated $\mathrm{ZBC}$ is expected, the activation energy yielding an estimate of the gap. In the high temperature phase, the activation energy obtained from the slope of our $\log (\mathrm{ZBC})$ data vs $1 / T$ is comparable to the activation energy estimated from the slope

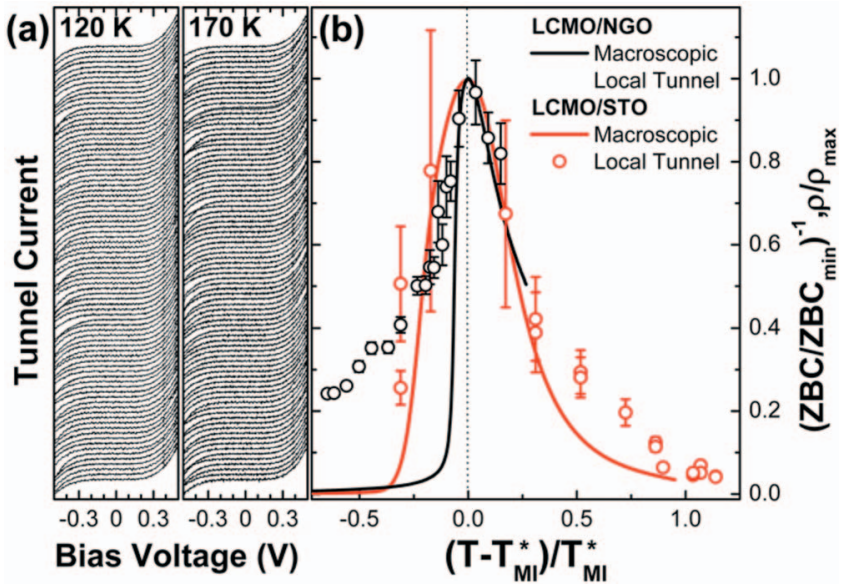

FIG. 2. (Color) (a) Local $I(V)$ curves acquired along a $350 \mathrm{~nm}$ trace on highly strained LCMO/STO for the metallic $(120 \mathrm{~K})$ and insulating $(170 \mathrm{~K})$ phases. (b) Local (open symbols) vs macroscopic (lines) electronic properties vs temperature for LCMO/NGO (black) and LCMO/STO (red). Temperatures are expressed relative to the respective insulator-to-metal transition temperatures: $T_{\mathrm{MI}}^{*}=T_{\mathrm{MI}}$ for resistivity curves, while for zero-bias conductance $T_{\mathrm{MI}}^{*}=T_{\mathrm{MI}}^{\mathrm{S}}$, the temperature at which ZBC reaches a minimum. Since scanning tunneling spectroscopy probes the DOS at the surface, $T_{\mathrm{MI}}^{\mathrm{S}}$ is slightly different to $T_{\mathrm{MI}}$. 
of $\log (\rho)$ vs $1 / T,(0.12 \pm 0.01) \mathrm{eV}$ for $\mathrm{LCMO} / \mathrm{STO}$ and $(0.07 \pm 0.01) \mathrm{eV}$ for LCMO/NGO. However, the thermal activation here may be only apparent. The computational study in Ref. 5 proposes the occurrence of dynamic nanoscale $\mathrm{AFM} / \mathrm{CO}$ correlations in the vicinity of the FM-AFM/CO phase boundary. If the characteristic lifetime of these correlations is shorter than the time scale of tunneling experiments, they will not be visualized in ZBC images. Nevertheless the presence of these correlations yields a finite $\operatorname{DOS}(\mu)$ at all temperatures for long Monte Carlo time scales in the calculation, ${ }^{5}$ with a temperature dependence that follows the trend of macroscopic conductivity. This is indeed observed in our films, suggesting that the observed activatedlike behavior does not come from a thermal excitation of carriers over a band gap but is due to dynamical AFM/CO correlations.

The DOS in Ref. 5 is not gapped above $T_{\mathrm{MI}}$, but presents a pseudogaplike depletion in the vicinity of $\mu$ with a pseudogap energy scale that globally decreases on cooling. We can directly test these predictions by calculating the normalized or logarithmic conductance, $(d I / d V) /(I / V)$, a method that attenuates the dependence on the tunneling barrier and yields a quantity proportional to the local DOS. ${ }^{20}$ Normalized conductance (NC) curves are presented in Fig. 3 for both highly (left panel) and weakly (right panel) strained films. At high temperatures NC curves present a depletion around zero bias. This depletion survives down to low temperatures, but NC peaks (in the case of LCMO/STO) or kinks (for LCMO/NGO) become increasingly marked upon cooling. These features have been previously ascribed to the spectral signature of polarons, ${ }^{2,21}$ the distance between the peaks being related to the polaron binding energy. In this context, a 'polaron' describes an object that is not just a charge coupled to a local lattice distortion, but which has a complex spin and orbital structure, rather like the AFM/CO correlations considered in Ref. 5.

An important result of Fig. 3 is that there is neither a discontinuity nor an abrupt change in the shape of spectra across the insulator-to-metal transition. No hard gap opens

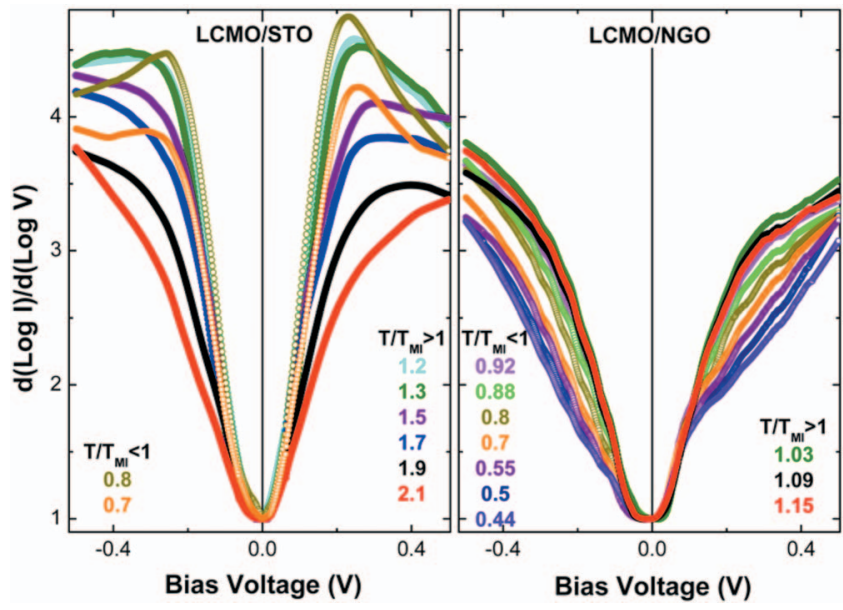

FIG. 3. (Color) Normalized conductance curves at different temperatures both above (full symbols) and below (open symbols) the insulator-to-metal transition for LCMO/STO (left panel) and LCMO/NGO (right panel). on warming through $T_{\mathrm{MI}}$ but the DOS is depleted in the vicinity of $\mu$ at all measured temperatures. However, subtle and gradual changes in the spectral shape are observed as a function of temperature. In the insulating phase, on cooling toward the transition, ZBC decreases as peaks/kinks develop at the flanks of the depletion, indicating a spectral weight transfer from low to high energies. On further cooling, in the metallic phase spectral weight increasingly builds up at the chemical potential and the height of conductance peaks slightly decreases, see Fig. 3(a). In terms of the findings of Ref. 5, these results can be interpreted as follows: In the insulating phase the AFM/CO correlation lifetime increases upon cooling, but decreases as ferromagnetic order sets in at $T<T_{\mathrm{MI}}$. Although the short lifetime of the correlations hindered us to directly image them in conductance maps, they are manifest through the pseudogappedlike depletion of tunneling conductance and the temperature evolution of the ZBC. It is important to note that the calculations of Ref. 5 were performed in the clean limit. Quenched disorder is known to enhance the colossal magnetoresistance and extends the region of the phase diagram where it occurs, ${ }^{22}$ but our results call for a systematic study of its effect on the AFM/CO correlations dynamics.

The width of the depletion or pseudogap, $\Delta$, was obtained for the curves in Fig. 3 as the half-distance between the two peaks (kinks) in the case of LCMO/STO(NGO) films. The pseudogap values are summarized in Fig. 4 for all samples. ${ }^{23}$ The behavior of $\Delta$ as a function of temperature is in qualitative agreement with the results of Ref. 5, globally increasing on warming. In the vicinity of $T_{\mathrm{MI}}$, the pseudogap energy changes its slope but $\Delta$ further increases in the hightemperature phase.

In conclusion, we have carried out a detailed analysis of the tunnel spectra of manganite films that sheds light into the puzzling relation between the local electronic properties and

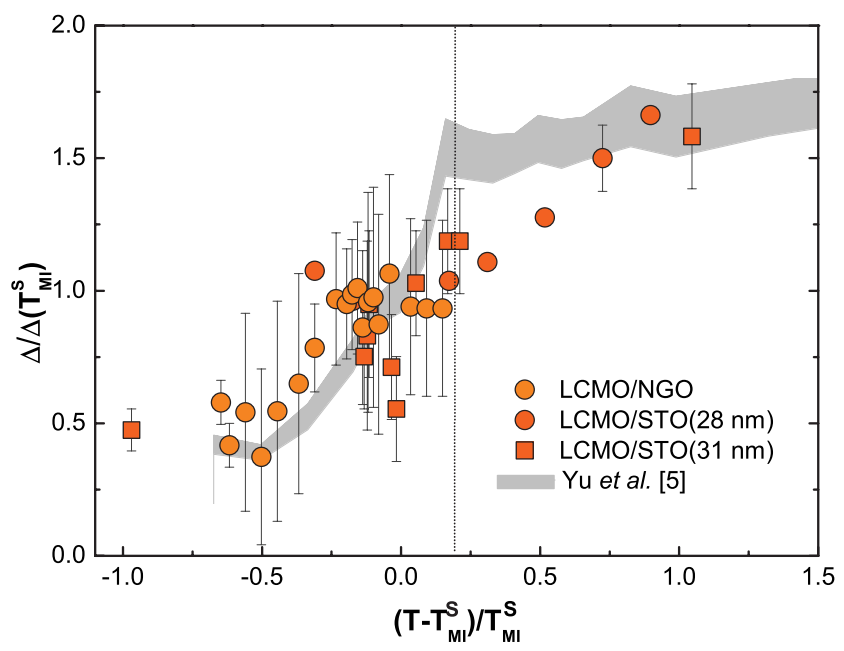

FIG. 4. (Color) Pseudogap energy (relative to its value at $T_{\mathrm{MI}}^{\mathrm{S}}$ ) vs reduced temperature for LCMO/STO in red (circles are data from this work, squares from Ref. 2), LCMO/NGO (orange) and results from the simulations in Ref. 5 (gray-shaded zone, covers the error). The temperatures are given relative to $T_{\mathrm{MI}}^{\mathrm{S}}$ for all experimental data (see caption of Fig. 2), and relative to the temperature of the local DOS minimum for the simulation. 
macroscopic transport across the insulator-to-metal transition. Although both the insulating and metallic phases present a pseudogapped normalized conductance, spectral weight redistributes as a function of temperature in such a way that the temperature evolution of the macroscopic conductivity is tracked by the density of states at the chemical potential. A similar behavior was predicted in Ref. 5, where nanoscale spin/charge correlations were found to be increasingly stable on cooling toward $T_{\mathrm{MI}}$ but their lifetime is reduced with the onset of ferromagnetic order. In addition, the predicted temperature evolution of the pseudogap energy $y^{5}$ is consistent with the measured $\Delta(T)$. This behavior is observed for highly and weakly strained films. Our results strongly support that dynamical spin/charge correlations play a preeminent role in the transport properties of colossal magnetoresistive manganites.

The authors thank Y. Rong and E. Dagotto for useful discussions and the Swiss National Science Foundation/MaNEP for financial support.
*Present address: Max Planck Institute for Chemical Physics of Solids, Nöthnitzer Str. 40, 01187 Dresden, Germany; seiro@cpfs.mpg.de

${ }^{\dagger}$ Present address: Instituto Balseiro and Centro Atómico Bariloche, Bustillo 9500, 8400 Bariloche, Argentina.

†Present address: Department of Physics, The Hong Kong University of Science \& Technology, Hong Kong.

${ }^{1}$ For a review, see, for example, Y. Tokura, Colossal Magnetoresistive Oxides (Gordon and Breach Science Publishers, Amsterdam, 2000); E. Dagotto,Nanoscale Phase Separation and Colossal Magnetoresistance (Springer-Verlag, Berlin, 2003); T. Chatterji, Colossal Magnetoresistive Manganites (Kluwer Academic Publishers, Dordrecht, Boston, London, 2004).

${ }^{2}$ S. Seiro, Y. Fasano, I. Maggio-Aprile, E. Koller, O. Kuffer, and O. Fischer, Phys. Rev. B 77, 020407(R) (2008).

${ }^{3}$ U. R. Singh, S. Chaudhuri, S. K. Choudhary, R. C. Budhani, and A. K. Gupta, Phys. Rev. B 77, 014404 (2008).

${ }^{4}$ H. M. Rønnow, C. Renner, G. Aeppli, T. Kimura, and Y. Tokura, Nature (London) 440, 1025 (2006).

${ }^{5}$ R. Yu, S. Dong, C. Şen, G. Alvarez, and E. Dagotto, Phys. Rev. B 77, 214434 (2008).

${ }^{6}$ S. Seiro, E. Koller, Y. Fasano, and Ø. Fischer, Appl. Phys. Lett. 91, 091913 (2007).

${ }^{7}$ M. Bibes, S. Valencia, L. Balcells, B. Martínez, J. Fontcuberta, M. Wojcik, S. Nadolski, and E. Jedryka, Phys. Rev. B 66, 134416 (2002).

${ }^{8}$ K.-H. Müller, K. Dörr, T. Walter, M. Sahana, K. Brand, and L. Schultz, J. Magn. Magn. Mater. 242-245, 447 (2002).

${ }^{9}$ B. Vengalis, A. Maneikis, F. Anisimovas, R. Butkutè, L. Dapkus, and A. Kindurys, J. Magn. Magn. Mater. 211, 35 (2000).

${ }^{10}$ J. H. Song, J.-H. Park, Y. H. Jeong, and T. Y. Koo, Physica B
312-313, 729 (2002).

${ }^{11}$ M. Ziese, H. C. Semmelhack, and K. H. Han, Phys. Rev. B 68, 134444 (2003).

${ }^{12}$ S. Valencia, L. Balcells, B. Martínez, and J. Fontcuberta, J. Appl. Phys. 93, 8059 (2003).

${ }^{13}$ L. Sudheendra, V. Moshnyaga, E. D. Mishina, B. Damaschke, Th. Rasing, and K. Samwer, Phys. Rev. B 75, 172407 (2007).

${ }^{14}$ S. Rößler, S. Ernst, B. Padmanabhan, S. Elizabeth, H. L. Bhat, F. Steglich, and S. Wirth, EPL 83, 17009 (2008).

${ }^{15}$ S. Seiro, Y. Fasano, I. Maggio-Aprile, O. Kuffer, and Ø. Fischer, J. Magn. Magn. Mater. 310, e243 (2007).

${ }^{16}$ S. Seiro, Ph.D. thesis 3995, University of Geneva, 2008.

${ }^{17}$ J. X. Ma, D. T. Gillaspie, E. W. Plummer, and J. Shen, Phys. Rev. Lett. 95, 237210 (2005).

${ }^{18}$ J. Mitra, M. Paranjape, A. K. Raychaudhuri, N. D. Mathur, and M. G. Blamire, Phys. Rev. B 71, 094426 (2005).

${ }^{19}$ In our experiments the apparent work function ranged between 1 and $1.5 \mathrm{eV}$.

${ }^{20}$ R. M. Feenstra, Phys. Rev. B 50, 4561 (1994).

${ }^{21}$ J. Y. T. Wei, N.-C. Yeh, and R. P. Vasquez, Phys. Rev. Lett. 79, 5150 (1997).

${ }^{22}$ E. Dagotto, S. Yunoki, C. Şen, G. Alvarez, and A. Moreo, J. Phys.: Condens. Matter 20, 434224 (2008).

${ }^{23}$ The energy scale of the depletion in our films, comparable to the activation energy in resistivity, is well below the $1.5 \mathrm{eV}$ observed by K. Fuchigami, Z. Gai, T. Z. Ward, L. F. Yin, P. C. Snijders, E. W. Plummer, and J. Shen, in Phys. Rev. Lett. 102, 066104 (2009) for the $\sqrt{2} \times \sqrt{2}$ reconstructed surface of a $\mathrm{La}_{5 / 8} \mathrm{Ca}_{3 / 8} \mathrm{MnO}_{3} / \mathrm{Nb}$-STO film. This indicates that the surface of our films has not undergone this kind of reconstruction. 